\title{
Temperature dependence of a vibrational exciton: Some methyl motions of durene*
}

\author{
Paras N. Prasad and Raoul Kopelman \\ Department of Chemistry, The University of Michigan, Ann Arbor, Michigan 48104
}

(Received 6 November 1972)

\begin{abstract}
An intramolecular vibrational frequency in a molecular crystal has been found to show a significant temperature dependence. The low lying intramolecular mode at $272 \mathrm{~cm}^{-1}$ (room temperature value) of durene- $h_{14}$ (and the corresponding durene- $d_{14}$ frequency at $242 \mathrm{~cm}^{-1}$ ) shows an increase of $\sim 9 \mathrm{~cm}^{-1}$ in frequency in going from room temperature to $100^{\circ} \mathrm{K}$. A theoretical discussion is presented on the role of various interactions that may give rise to the temperature dependence of the intramolecular mode. The theoretical considerations suggest that, although the thermal expansion of the lattice may be contributing to this temperature dependence, the dominant contribution is due to the cubic anharmonic interactions involving methyl torsions. It is suggested that in complex molecular crystals, where there are some very low lying intramolecular modes, the distinction between lattice modes and intramolecular modes based on the criterion that only lattice modes show frequency shift with temperature may be misleading. In such cases our mixed crystal criterion that the phonons obey the amalgamation limit in isotopic (H-D) mixed crystals, whereas intramolecular vibrations are in the separated band limit, should be more reliable. This is clearly demonstrated in the case of durene.
\end{abstract}

\section{INTRODUCTION}

The problem of vibrations in molecular solids is usually simplified by the assumption of the separability of the external modes from the internal modes. ${ }^{1-4}$ The external modes (or the lattice vibrations) are the solutions for the motion of the molecule as a whole under the intermolecular interaction. The calculation of such lattice modes are made on the assumption that the molecules vibrate internally as semirigid bodies. ${ }^{4-8}$ Internal modes (or the intramolecular vibrations), on the other hand, are obtained by solving for the internal motions within the molecule, considering that these motions are subject to a strong valence field in the first approximation. Allowance for the excitation exchange among the identical molecules causes the splitting of the intramolecular vibration into an exciton band. ${ }^{9-13}$ In a real system of molecular solids, however, there exist finite interactions between the lattice modes and the intramolecular modes. These interactions show many manifestations in the vibrational spectra of molecular solids. Line broadening and combination bands involving lattice vibrations are some of the manifestations that have been found in the spectra of the intramolecular vibrations. ${ }^{14}$ Acoustic attenuation is another example which has been observed ${ }^{15-17}$ in the ultrasonic spectrum of some molecular solids.

There have been some attempts ${ }^{3,15,18}$ to treat the above problem theoretically, but such treatments are not yet complete and a widespread understanding of these interactions still awaits further experimental and theoretical work. In the present paper we present some experimental results which display a very different manifestation of the interaction between lattice modes and intramolecular modes. The coupling between the intramolecular vibrations and the lattice vibrations can make the frequency of the intramolecular mode temperature dependent. Here we report on such a temperature dependence observed in the durene crystal. In all previous studies only lattice mode frequencies have been found to show a significant temperature dependence..$^{1920}$ As a matter of fact, this temperature dependence of the lattice modes has often been used ${ }^{20}$ as a criterion to distinguish them from the intramolecular modes.

We also present a theoretical description in which we discuss the role of various interactions that may give rise to the temperature dependence of an intramolecular mode. The experimental study of the crystal shift and its temperature study permits us to comment on the relative roles of the various interactions that may give rise to the temperature effect. The theoretical considerations suggest that although the thermal expansion of the lattice may be contributing to this temperature dependence, the dominant contribution is due to the cubic anharmonic interactions involving methyl torsions.

\section{THEORETICAL}

We start with the intramolecular vibrational Hamiltonian of the free molecule and then generate the corresponding Hamiltonian for the crystal. This approach is desirable in the present context, as we wish to consider the role of the crystal shift (gas to crystal phase shift) term in giving rise to the temperature dependence of the frequency of an intramolecular vibration. The intramolecular vibrational Hamiltonian for the free molecule is given as

$$
H=\frac{1}{2} \sum_{j}\left(P_{j}^{2}+\omega_{j}^{2} Q_{j}^{2}\right),
$$

where $Q_{j}$ is the normal coordinate for the $j$ th mode and $P_{j}$ the corresponding momentum coordinate. $\omega_{j}$ is the 
eigenfrequency. The transition to quantum mechanics and is achieved by the following:

$$
\begin{aligned}
& Q_{j}=\left(2 \omega_{j}\right)^{-1 / 2}\left(B_{j}+B_{j}^{+}\right), \\
& P_{j}=i\left(\omega_{j} / 2\right)^{1 / 2}\left(B_{j}-B_{j}^{+}\right),
\end{aligned}
$$

where $B_{j}^{+}$and $B_{j}$ are, respectively, the creation and annihilation operators for the mode $j$. This is, in atomic units, $(\hbar=1)$. With this substitution the Hamiltonian takes the form

$$
H=\sum_{j} \omega_{j}\left(B_{j}{ }^{+} B_{j}+\frac{1}{2}\right) .
$$

In the solid state the molecule is subject to an additional potential field, the crystal field $V^{c}$. It is given as

$$
V^{c}=\sum_{n \alpha} V_{n \alpha^{c}}=\sum_{n \alpha>n} \sum_{n_{1} \alpha_{1}} V_{n \alpha, n_{1} \alpha_{1}^{c}},
$$

where $n$ and $n_{1}$ label the primitive unit cell of the crystal while $\alpha$ and $\alpha_{1}$ label the molecular site in the unit cell. In the crystal the Hamiltonian corresponding to intramolecular vibrations becomes

$$
\begin{aligned}
H=\sum_{j} \sum_{n \alpha}\left(\omega_{j}+D_{j n \alpha}\right)\left(B_{j n \alpha}+B_{j n \alpha}+\frac{1}{2}\right) \\
\quad+\sum_{j} \sum_{n \alpha>n 1 \alpha_{1}} M_{j, n \alpha, n \mid \alpha_{1}}\left(B_{j n \alpha}+B_{j n 1 \alpha_{1}}+B_{j n_{1} \alpha_{1}}+B_{j n \alpha}\right),
\end{aligned}
$$

where the term $D_{j n a}$ is the band shift term and is given as

$$
D_{j n \alpha}=\left\{V_{j j, n \alpha}+\sum_{j^{\prime} \neq j}\left[V_{j j^{\prime}, n \alpha} V_{j^{\prime} j, n \alpha} /\left(E_{j}-E_{j^{\prime}}\right)\right]+\cdots\right\}
$$

with

$$
V_{j j^{*}, n \alpha}=\left(4 \omega_{j j} \omega_{j^{\prime}}\right)^{-1 / 2}\left(\partial^{2} V^{c} / \partial Q_{j n \alpha} \partial Q_{j^{\prime} n \alpha}\right)_{Q=Q_{0}} .
$$

Unless the molecular geometry is distorted in the crystal, the leading term is $V_{j j, n \alpha}$. In our system (durene) this seems to be the case ${ }^{21}$ and thus we approximate $D_{j n \alpha}$ by $V_{j i, n \alpha}$. The band splitting term is

$$
\begin{aligned}
M_{j, n \alpha, n_{1} \alpha_{1}} & =\left\{V_{j n \alpha, j n_{1} \alpha_{1}}\right. \\
& \left.+\sum_{j \neq j}\left[V_{j n \alpha_{1}, j_{1} n_{1} \alpha_{1}} V_{j n \alpha, j_{1} n_{1} \alpha_{1}} /\left(E_{j}-E_{j_{1}}\right)\right]\right\}
\end{aligned}
$$

and

$$
V_{j n \alpha, j_{1 n} \alpha_{1}}=\left(4 \omega_{j} \omega_{j_{1}}\right)^{-1 / 2}\left(\partial^{2} V^{c} / \partial Q_{j n \alpha} \partial Q_{j_{1 n} \alpha_{1}}\right)_{Q=Q_{0}},
$$

where the interaction $V_{j n \alpha, j n_{1} \alpha_{1}}$ is the excitation exchange interaction for the mode $j$, while $V_{j n^{\alpha}, j_{1 n_{1} \alpha_{1}}}$ is the intermolecular configuration interaction between the modes $j$ and $j_{1}$. We approximate $M_{j, n \alpha_{n} n_{1} \alpha_{1}}$ by

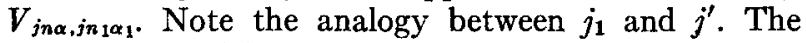
Hamiltonian (5) is diagonalized by the following transformation in the restricted Frenkel ${ }^{12}$ limit:

$$
B_{j n \alpha}=N^{-1 / 2} \sum_{\mathbf{k}, f} A_{\alpha f} B_{j \mathbf{k} f} \exp \left(i \mathbf{k} \cdot \mathbf{X}_{n \alpha}\right)
$$

$$
B_{j n \alpha}{ }^{+}=N^{-1 / 2} \sum_{\mathbf{k}, f} A_{\alpha f} B_{j \mathrm{kf}}{ }^{+} \exp \left(-i \mathbf{k} \cdot \mathbf{X}_{n \alpha}\right) .
$$

Here $\mathrm{X}_{n \alpha}$ is the position vector of the site $n \alpha . A_{\alpha f}$ represents the $\alpha, f$ element of the interchange group transformation with $f$ labeling the interchange group states. For a monoclinic crystal like naphthalene or durene the coefficient is real. $B_{j \mathbf{k} f}{ }^{+}$and $B_{j \mathbf{k} f}$ are, respectively, the creation and annihilation operators for the exciton state $|j \mathbf{k} f\rangle$ with wave vector $\mathbf{k}$. The Hamiltonian (5) assumes the form

$$
H_{\mathrm{ex}}=\sum_{j, \mathbf{k}, f} \omega_{j \mathrm{k} f}\left(B_{j \mathrm{k} f}+B_{j \mathrm{k} f}+\frac{1}{2}\right) .
$$

From Eqs. (2) and (10) we can see that

$$
\begin{aligned}
Q_{j n \alpha}=\left(2 \omega_{j} N\right)^{-1 / 2} \sum_{\mathbf{k}, f}\left(B_{j \mathrm{k} f}+B_{j(-\mathrm{k}) f^{+}}\right) A_{\alpha f} \\
\times \exp \left(i \mathbf{k} \cdot \mathbf{X}_{n \alpha}\right)
\end{aligned}
$$$$
\text { or }
$$

where

$$
Q_{i n \alpha}=\left(2 \omega_{j} N\right)^{-1 / 2} \sum_{\mathbf{k}, f} \Phi_{j \mathbf{k} f} A_{\alpha, f} \exp \left(i \mathbf{k} \cdot \mathbf{X}_{n \alpha}\right),
$$

$$
\Phi_{j \mathrm{k} f}=B_{j \mathrm{k} f}+B_{j(-\mathrm{k}) f^{+}} .
$$

The phonon Hamiltonian is described as $^{22}$

$$
H_{\mathrm{ph}}=\sum_{\mathrm{q}, S} \omega_{\mathrm{q} S}\left(b_{\mathrm{q} S}+b_{\mathrm{q} S}+\frac{1}{2}\right) .
$$

Here, $\omega_{\mathbf{q} S}$ is the energy of a phonon of wave vector $\mathbf{q}$ and branch $S$. There are twelve phonon branches in our monoclinic crystal. $b_{\mathrm{q} S}{ }^{+}$and $b_{\mathrm{q} S}$ are, respectively, the creation and annihilation operators for the phonon $|\mathrm{q} S\rangle$. The molecular displacement operators $R_{\text {un } \alpha}$ are given $\operatorname{as}^{4}$

$$
R_{u n \alpha}=\sum_{\mathbf{q}, S}\left(2 N M \omega_{\mathbf{q}, S}\right)^{-1 / 2} \eta_{\alpha}{ }^{u}(\mathbf{q} S) \exp \left(i \mathbf{q} \cdot \mathbf{X}_{n}\right) \Phi_{\mathbf{q}, S}
$$

where

$$
\Phi_{\mathrm{q}, S}=\left(b_{\mathrm{q} S}+b_{-\mathrm{q} S}{ }^{+}\right) .
$$

In Eq. (15), $u$ labels the six degrees of molecular freedom for a nonlinear molecule. The branch vectors $\eta_{\alpha}{ }^{\mu}(\mathrm{q} S)$ are normalized by the following relation ${ }^{4}$ :

$$
\sum_{\alpha, u, u^{\prime}} \eta_{\alpha^{u}}(\mathbf{q}, S) m_{u u^{\prime}, \alpha} \eta_{\alpha^{u}}{ }^{\prime}\left(\mathbf{q}, S^{\prime}\right)=M \delta_{S, S^{\prime}}
$$

where $M_{u u^{\prime}, \alpha}$ is the mass coefficient for the molecule at site $\alpha$ corresponding to the coordinates $u$ and $u^{\prime}$.

The interactions between the vibrations can be divided into two classes:

(1) Harmonic interaction between the intramolecular vibrations and the lattice vibrations. This arises because of the nonrigidity of the molecules. Under the rigid body approximation we ignore the following force 
constant term:

$$
H_{h}=\frac{1}{2} \sum_{j, n \alpha ; u, n_{1} \alpha_{1}}\left(\partial^{2} V^{c} / \partial Q_{j n \alpha} \partial R_{u n_{1} \alpha_{1}}\right)_{0} Q_{j n \alpha} R_{u n 1 \alpha_{1}} \cdot
$$

We call $H_{h}$ the harmonic perturbation. Using the expansion (12) for $Q_{j n \alpha}$ and (15) for $R_{\text {un } 1 \alpha_{1}}$ we get $H_{h}=\frac{1}{2} \sum_{j, \mathbf{k}, f} \sum_{\mathbf{q}, S} \sum_{n \alpha} \sum_{n_{1} \alpha_{1} u} \sum N^{-1}\left(4 \omega_{j} \omega_{\mathbf{q}, S} M\right)^{-1 / 2} \eta_{\alpha_{1}}{ }^{u}(\mathbf{q} S) \exp \left(i \mathrm{q} \cdot \mathbf{X}_{n_{1}}\right) A_{\alpha f}$

$$
X \exp \left(i \mathbf{k} \cdot \mathbf{X}_{n \alpha}\right) \Phi_{j \mathbf{k} f} \Phi_{\mathrm{q} S}\left(\partial^{2} V^{c} / \partial Q_{j n \alpha} \partial R_{u n_{1} \alpha_{1}}\right)_{0}
$$

or

$$
H_{h}=\sum_{j, \mathrm{k}, f} \sum_{\mathbf{q}, S} V_{j \mathrm{k} f, \mathrm{q} S} \Phi_{j \mathrm{k} f} \Phi_{\mathrm{q} S},
$$

where

$$
V_{j \mathbf{k} f, \mathbf{q} S}=(2 N)^{-1} \sum_{n \alpha} \sum_{n_{1} \alpha_{1}} \sum_{\mathrm{u}}\left(4 \omega_{j} \omega_{\mathrm{q} S} M\right)^{-1 / 2} \eta_{\alpha_{1}}{ }^{u}(\mathbf{q} S) A_{\alpha f} \exp \left(i \mathbf{q} \cdot \mathbf{X}_{n_{1}}+i \mathbf{k} \cdot \mathbf{X}_{n \alpha}\right)\left(\partial^{2} V^{c} / \partial Q_{j n \alpha} \partial R_{u n_{1} \alpha_{1}}\right)_{0}
$$

The momentum conservation requires $\mathbf{k}+\mathbf{q}=\mathbf{g}$, where $\mathbf{g}$ is a reciprocal lattice vector. From this place onward we use $I$ to represent the set of three quantum numbers $j, \mathbf{k}, f$ for the intramolecular vibrations, while $L$ represents the lattice quantum numbers q, $S$. In a general case $\theta=I$ or $L$. This interaction gives rise to a self-energy $\Delta_{I}^{h}$ for the intramolecular vibration $I . \Delta_{I}{ }^{h}$ can be shown to be (see the Appendix for the derivation)

$$
\begin{aligned}
\Delta_{I}^{h} & =\sum_{L} V_{I L} V_{L I}\left\{\left[P /\left(\omega-\omega_{L}\right)\right]-\left(\omega+\omega_{L}\right)^{-1}\right\} \\
& \approx \sum_{L} V_{I L} V_{L I}\left\{\left[P /\left(\omega_{I}-\omega_{L}\right)\right]-\left(\omega_{I}+\omega_{L}\right)^{-1}\right\},
\end{aligned}
$$

where $P$ ensures that the term vanishes when the denominator is zero and is unity otherwise. $\Delta_{I}^{h}$ is independent of the phonon occupation number and so the temperature dependence of $\Delta_{I}^{h}$ does not come from the temperature variation of the phonon occupation number. However, the phonon frequencies $\omega_{L}$ are temperature dependent due to lattice anharmonicity and this makes $\Delta_{I}{ }^{h}$ temperature dependent. Because of this indirect temperature dependence the frequency of the intramolecular mode $I \omega_{I}^{h}=\omega_{I}+\Delta_{I}^{h}$ would decrease with increase in temperature, as $\omega_{L}$ decreases with increasing temperature.

(2) Anharmonic interactions include third and other higher order force constant terms. We consider only the cubic and quartic terms. The anharmonic interaction $H_{A}$ can then be expressed as

$$
\begin{aligned}
H_{A}=\sum_{\theta_{1}} \sum_{\theta_{2}} \sum_{\theta_{3}} V_{\theta_{1} \theta_{2} \theta_{3}} \xi_{\theta_{1}} \xi_{\theta_{2}} \xi_{\theta_{3}} \\
\\
+\sum_{\theta_{1}} \sum_{\theta_{2}} \sum_{\theta_{3}} \sum_{\theta_{4}} V_{\theta_{1} \theta_{2 \theta_{3} \theta_{4}} \xi \theta_{1}} \xi_{\theta_{2}} \xi_{\theta_{3}} \xi_{\theta_{4}},
\end{aligned}
$$

where

$$
\begin{aligned}
\xi_{\theta} & =R & & \text { for } \theta=L \\
& =Q & & \text { for } \theta=I .
\end{aligned}
$$

The anharmonic interactions can be divided into three classes. (a) lattice anharmonicity: for this $\theta_{1}, \theta_{2}, \theta_{3}$, and $\theta_{4}$ are $L_{1}, L_{2}, L_{3}$, and $L_{4}$; (b) internal anharmonicity: in this case $\theta_{1}, \theta_{2}, \theta_{3}$, and $\theta_{4}$ are $I_{1}, I_{2}, I_{3}$, and $I_{4} ;(\mathrm{c})$ mixed mode anharmonicity: this is described by the terms in which $\theta_{1}, \theta_{2}, \theta_{3}$, and $\theta_{4}$ involve both $I$ and $L$. The lattice contribution to the temperature dependence of a vibrational exciton comes from the mixed mode anharmonicity. This contribution can be divided in two classes: (i) The exciton-phonon coupling which exists even in a lattice without thermal expansion property. This coupling arises because of the variations in the crystal potential (for the vibrational exciton) with the arbitrary molecular displacements during the period of lattice vibrations. (ii) The contribution due to the thermal expansion of the lattice. This is another manifestation of the lattice anharmonicity. The thermal expansion gives rise to a homogeneous displacement of the molecules in the crystal with a change in the temperature. This displacement changes the lattice parameters. As a result the crystal potential changes which in turn changes the frequency of a vibrational exciton. We would like to point out that only the anharmonic interaction of class (i) gives rise to an imaginary contribution to the self-energy (vide infra) and thus to the line broadening of a vibrational exciton transition (and also a phonon transition). The anharmonic manifestation of class (ii) leads only to a frequency shift but not to line broadening. ${ }^{23}$

The total molecular displacement $R_{u n \alpha}$ for a lattice with thermal expansion property is given as

$$
R_{u n \alpha}=R_{u n \alpha^{0}}+\sum_{u^{\prime}} \epsilon_{u u^{\prime}} X_{u^{\prime} n \alpha} .
$$

In this equation $R_{u n \alpha^{0}}$ is the arbitrary molecular displacement which exists even in the lattice without thermal expansion. It is this part of the $R_{u n \alpha}$ which has been considered in the harmonic approximation theory discussed earlier. This displacement $R_{u n \alpha}{ }^{0}$ describes the contribution of class (i). The second term on the right hand side of Eq. (24) describes the homogeneous displacement in the crystal due to the thermal expansion. In this term $\epsilon_{u u^{\prime}}$ is a component of the symmetric strain tensor. $X_{u^{\prime} n \alpha}$ is the $u^{\prime}$ component of the position vector $\mathbf{X}_{n \alpha}$. The thermal expansion contribution involves this part of the displacement operator $R_{\text {un } \alpha}$. We consider first the interactions in a lattice without thermal expansion. In this case the molecular displacement is approximated by $\boldsymbol{R}_{u n \alpha^{0}}$, which is defined by 
Eq. (15) in terms of the phonon operator. For this case a mixed mode anharmonic coupling function $V_{I L_{1} L_{2}}$ is defined as

$$
\begin{aligned}
& V_{I L_{1} L_{2}}=\sum_{n \alpha, n_{1} \alpha_{1} u_{1}, n_{2 \alpha} \alpha_{2} u_{2}}(3 !)^{-1}(N)^{-8 / 2}\left(8 \omega_{j} \omega_{q_{1} S_{1}} \omega_{q_{2} S_{2}} M^{2}\right)^{-1 / 2} \eta_{\alpha_{1}}{ }^{{ }_{1}}\left(S_{1} \mathrm{q}_{1}\right) A_{\alpha f} \eta_{\alpha_{2}}{ }^{u_{2}}\left(S_{2} \mathrm{q}_{2}\right) \\
& \times \exp \left[i\left(\mathbf{k} \cdot \mathbf{X}_{n \alpha}+\mathbf{q}_{1} \cdot \mathbf{X}_{n_{1}}+\mathbf{q}_{2} \cdot \mathbf{X}_{n_{2}}\right)\right]\left(\partial^{3} V / \partial Q_{j n \alpha} \partial R_{u_{1 n} \alpha_{1}{ }_{1}^{0}} \partial R_{u 2 n 2 \alpha_{2}}\right)_{0},
\end{aligned}
$$

where

$$
I=j, \mathbf{k}, f, \quad L_{1}=\mathrm{q}_{1}, S_{1}, \quad \text { and } \quad L_{2}=\mathrm{q}_{2}, S_{2} \text {. }
$$

The momentum conservation requires $\mathbf{k}+\mathbf{q}_{1}+\mathbf{q}_{2}=\mathbf{g}$, where $\mathbf{g}$ is a reciprocal lattice vector. Other coupling functions are defined in a similar way. The self-energy $\Delta_{I} A$ of an intramolecular mode $I$ due to the anharmonic interactions in a lattice without thermal expansion is given $\mathbf{a s}^{24}$

$$
\begin{aligned}
\Delta_{I}^{A}=\sum_{\theta_{1}} \sum_{\theta_{2}} 18\left[V_{I \theta_{1} \theta_{2}}{ }^{2}\left(\frac{\left(1+n_{\theta_{1}}+n_{\theta_{2}}\right) P}{\omega_{I}-\omega_{\theta_{1}}-\omega_{\theta_{2}}}+\frac{2\left(n_{\theta_{1}}-n_{\theta_{2}}\right) P}{\omega_{I}+\omega_{\theta_{1}}-\omega_{\theta_{2}}}-\frac{\left(1+n_{\theta_{1}}+n_{\theta_{2}}\right)}{\omega_{I}+\omega_{\theta_{1}}+\omega_{\theta_{2}}}\right)-2 V_{I I \theta_{1}} V_{\theta_{1} \theta_{2} \theta_{2}} \frac{\left(2 n_{\theta_{2}}+1\right)}{\omega_{\theta_{1}}}\right] \\
+\sum_{\theta} 12 V_{I I \theta \theta}\left(2 n_{\theta}+1\right) .
\end{aligned}
$$

We see that this self-energy is temperature dependent mainly because of the presence of the occupation number $n_{\theta}$. As the temperature dependence of $n_{\theta}$ is large, this temperature dependence should dominate over the one due to $\Delta_{I}{ }^{h}$, which is independent of $n_{\theta}\left(=n_{L}\right)$. As the occupation number $n_{I}$ for an intramolecular mode $I$ is small, compared to $n_{L}$ of a lattice mode $L$, and also because the internal anharmonicity coupling is small, compared to both mixed mode anharmonic coupling and lattice anharmonic coupling, we expect that the terms involving mixed mode anharmonicity and lattice anharmonicity should make the major contribution. In view of the above discussion, we have

$$
\begin{aligned}
\Delta_{T}^{A}=\sum_{L_{1}} \sum_{L_{2}} 18\left[V_{I L_{1} L_{2}}{ }^{2}\left(\frac{\left(1+n_{L_{1}}+n_{L_{2}}\right) P}{\omega_{I}-\omega_{L_{1}}-\omega_{L_{2}}}+\frac{2\left(n_{L_{1}}-n_{L_{2}}\right) P}{\omega_{I}+\omega_{L_{1}}-\omega_{L_{2}}}-\frac{\left(1+n_{L_{1}}+n_{L_{2}}\right)}{\omega_{I}+\omega_{L_{1}}+\omega_{L_{2}}}\right)-2 V_{I I L_{1}} V_{L_{1} L_{2} L_{2}} \frac{\left(2 n_{L_{2}}+1\right)}{\omega_{L_{1}}}\right] \\
+\sum_{L} 12 V_{I I L L}\left(2 n_{L}+1\right) .
\end{aligned}
$$

Let us examine the physical nature of the different coupling functions contained in Eq. (27). $V_{I L_{1} L_{2}}$ is defined by Eq. (25), in which the term

$$
\left(\partial^{3} V / \partial Q_{j n \alpha} \partial R_{u_{1 n} \alpha_{1}}{ }^{0} \partial R_{u_{2 n} \alpha_{2}}{ }^{0}\right)_{0} \approx\left\{\left(\partial / \partial Q_{j n \alpha}\right)\left(\partial^{2} V^{c} / \partial R_{u_{1 n} \alpha_{1} \alpha_{1}}{ }^{0} \partial R_{u 2 n 2 \alpha_{2}}\right)_{R=R_{0}}\right\}_{Q=Q_{0}}
$$

(where $R_{0}$ and $Q_{0}$ are the equilibrium positions of the inter- and intramolecular modes, respectively) describes the variation of the intermolecular forces with intramolecular displacements. ${ }^{25}$ Site splitting studies of Hochstrasser and Prasad $^{26}$ suggest the dependence of intermolecular forces on intramolecular motions, but, in view of the fact that their result also fits another explanation, the importance of such coupling is not established. However, considering the small displacements involved in the intramolecular vibrations (this may not be true for a torsional motion arising from hindered rotation, and, perhaps for some very low lying modes - see below) one would expect a small contribution from the coupling functions $V_{I L_{1} L_{2}}$. The contribution to $\Delta_{T}^{A}$ from $V_{I L_{1} L_{2}}$ can be negative or positive, depending on the frequency $\omega_{T}$ of the intramolecular vibrations relative to the lattice vibrations. In other words, this interaction may lead to an increase or decrease of the frequencies with an increase in temperature depending on whether $\omega_{I}<2 \omega_{L}$ or $\omega_{I}>2 \omega_{L}$, where $\omega_{L}$ is the maximum phonon frequency.

The coupling function $V_{L_{1} L_{2} L_{2}}$ is the lattice anharmonicity. The coupling function $V_{I I L_{1}}$ is given as

$$
\begin{aligned}
V_{I I L_{1}}=\sum_{n \alpha} \sum_{n^{\prime} \alpha^{\prime}} \sum_{n \alpha_{1} \alpha_{1} u_{1}}\left\{(3 !)^{-1}(N)^{-3 / 2}\left(8 \omega_{j}^{2} \omega_{\mathbf{q}_{1} S_{1}} M\right)^{-1 / 2} \eta_{\alpha_{1}}{ }^{u_{1}}\left(S_{1} \mathbf{q}_{1}\right) A_{\alpha^{\prime} f} A_{\alpha f}\right. \\
\\
\left.\times \exp \left[i\left(\mathbf{q}_{1} \cdot \mathbf{X}_{n_{1}}+\mathbf{k} \cdot \mathbf{X}_{n \alpha}+\mathbf{k} \cdot \mathbf{X}_{n^{\prime} \alpha^{\prime}}\right)\right]\left(\partial^{3} V / \partial Q_{j n \alpha} \partial Q_{n^{\prime} \alpha^{\prime}} \partial R_{u_{1} n_{1} \alpha_{1}}{ }^{0}\right)_{0}\right\} .
\end{aligned}
$$

In Eq. (29) we have used $I=(j, \mathbf{k}, f)$ and $L_{\mathbf{l}}=\left(\mathbf{q}_{1}, S_{1}\right)$. Also,

$$
\left(\partial^{3} V / \partial Q_{j n \alpha} \partial Q_{j n^{\prime} \alpha^{\prime}} \partial R_{u n_{1} \alpha_{1}}{ }^{0}\right)_{0} \approx\left\{\left(\partial / \partial R_{u 1 n_{1 \alpha_{1}}}{ }^{0}\right)\left(\partial^{2} V^{c} / \partial Q_{j n \alpha} \partial Q_{j n^{\prime} \alpha^{\prime}}\right)_{Q=Q_{0}}\right\}_{R=R_{0}}
$$

It can be seen from Eq. (30) that $V_{I I L_{1}}$ consists of the terms

$$
\sum_{u_{1 n_{1} \alpha_{1}}}\left\{\left(\partial / \partial R_{u_{1} n_{1} \alpha_{1}}\right)\left(D_{j n \alpha}\right)\right\}_{R=R_{0}} \quad \text { and } \sum_{u_{1} n_{1} \alpha_{1}}\left\{\left(\partial / \partial R_{u_{1} n_{1} \alpha_{1}}{ }^{0}\right)\left(M_{j, n \alpha, n^{\prime} \alpha^{\prime}}\right)\right\}_{R=R_{0}}
$$

which describe the variations of the band shift term and splitting term (excitation exchange), respectively, with the intermolecular displacements. $V_{I I L_{1}}$ can, thus, be written as

$$
V_{I I L_{1}}=D_{I, L_{1}}+M_{I, L_{1}}
$$

where $D_{r, L_{1}}$ and $M_{I, L_{1}}$ involve, respectively, the derivatives of the shift $(D)$ term and the splitting $(M)$ terms with respect to an intermolecular displacement $R$. These terms are, respectively, analogous to the dispersion force 
exciton-phonon coupling $\left[D_{S}(\mathbf{q})\right]$ and the resonance force exciton-phonon coupling $\left[M_{S}(\mathbf{k}, \mathbf{q})\right]$ of a Frenkel electronic exciton with a phonon. ${ }^{27}$

In light of the above discussion the temperature dependence of $\omega_{r}^{A}=\left(\omega_{I}+\Delta_{Y}^{A}\right)$ due to the term [Eq. (27)] $-36 V_{I I L_{1}} V_{L_{1} L_{2} L_{2}}\left[\left(2 n_{L_{2}}+1\right) / \omega_{L_{1}}\right]$ of the self-energy $\Delta_{I} A$ can be regarded as arising from the variation in the $D$ shift and/or $M$ term with the molecular motion in the lattice. The sign of the temperature dependence is determined by the signs of $V_{I I L_{1}}$ and $V_{L_{1} L_{2} L_{2}}$. The frequency $\omega_{I}{ }^{A}$ decreases with temperature if $V_{I I L_{1}}$ and $V_{L_{1} L_{2} L_{2}}$ have the same sign. This is, usually, the case if the $D$-shift term and the $M$ terms are both positive. ${ }^{28}$ On the other hand, if the $D$-shift term and the $M$ terms are both negative (or the dominant of the two is negative), in this case $V_{I I L_{1}}$ and $V_{L_{1} L_{2} L_{2}}$ have opposite signs and an increase of frequency with temperature should be observed. The quartic anharmonicity term $V_{I I L L}$ consists of the terms

$$
\sum_{u_{1} n_{1} \alpha_{1}, u_{2 n} \alpha_{2}}\left\{\left(\partial^{2} / \partial R_{u_{1} n_{1} \alpha_{1}}{ }^{2} \partial R_{u_{2 n} \alpha_{2}}\right)\left(D_{j n \alpha}\right)\right\}_{R=R_{0}} \quad \text { and } \quad \sum_{u_{1} n_{1} \alpha_{1}, u_{2 n} \alpha_{2}}\left\{\left(\partial^{2} / \partial R_{u_{1} n_{1} \alpha_{1}}{ }^{0} \partial R_{u_{2 n} \alpha_{2}}{ }^{0}\right)\left(M_{j, n \alpha, n^{\prime} \alpha^{\prime}}\right)\right\}_{R=R_{0}} \text {. }
$$

The quartic anharmonicity term is thus analogous to the quadratic exciton(electron)-phonon interaction which arises because of the change in the phonon eigenstate description (normal coordinate) during the excitonic transition. The contribution due to quartic anharmonicity should have the same sign as that of the gas to crystal frequency shift of the intramolecular mode $I .^{29}$

In systems like durene, there are some low lying torsional motions of the methyl groups. These torsional motions deserve special consideration. Although these modes are intramolecular in origin, very often the barrier hindering the methyl rotation may be to a large extent intermolecular. For this reason they show many similarities with the phonons. The torsions show line broadening features similar to that of the phonons. ${ }^{30}$ This shows that these motions are very anharmonic. In view of this, it appears that these motions can play an important role in the anharmonic coupling. However, their contribution follows a description very similar to that for the phonons and the expression for the self-energy due to their contribution is given from Eq. (27) with $L$ substituted by $T$ (representing a torsion). Coupling functions $V_{I T T}, V_{I I T}$ (and also mixed modes functions $V_{I T L}$, etc.) are derived in a way analogous to that for $V_{I L L}, V_{I I L}$.

Next we would like to consider the contribution due to thermal expansion of the lattice. ${ }^{31}$ We expand the $D$ and $M$ terms in a Taylor series in the homogeneous displacement due to thermal expansion. This displacement is given by the second term on the right hand side of Eq. (24). If we retain only up to the linear term of the Taylor series, we have

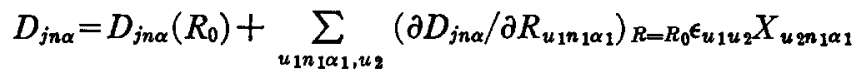

and

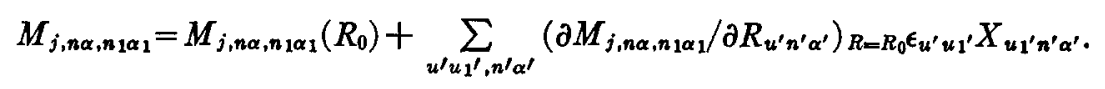

The total vibrational Hamiltonian (5) can be rewritten for a lattice with thermal expansion as

where

$$
H=H\left(R_{0}\right)+H(\epsilon)
$$

$$
H\left(R_{0}\right)=\sum_{j n \alpha}\left[\omega_{j}+D_{j n \alpha}\left(R_{0}\right)\right]\left(B_{j n \alpha}+B_{j n \alpha}+\frac{1}{2}\right)+\sum_{j} \sum_{n \alpha>n_{1} \alpha_{1}} M_{j, n \alpha_{1} n_{1} \alpha_{1}}\left(R_{0}\right)\left(B_{j n \alpha}+B_{j n_{1 \alpha}}+B_{j n_{1} \alpha_{1}}+B_{j n \alpha}\right)
$$

and

$$
\begin{aligned}
& H(\epsilon)=\sum_{j}\left\{\sum_{n_{1} \alpha_{1}} \sum_{u_{1} u_{2}}\left(\partial D_{j n \alpha} / \partial R_{u_{1 n} \alpha_{1}}\right)_{R=R_{0} \epsilon_{u_{1} u_{2}}} X_{u_{2 n 1 \alpha_{1}}}\right\}\left(B_{j n \alpha}+B_{j n \alpha}+\frac{1}{2}\right) \\
& +\sum_{j} \sum_{n \alpha>n_{1} \alpha_{1}}\left\{\sum_{n^{\prime} \alpha^{\prime}} \sum_{u^{\prime} u_{1^{\prime}}}\left(\partial M_{j, n \alpha, n \alpha_{1} \alpha_{1}} / \partial R_{u^{\prime} n^{\prime} \alpha^{\prime}}\right)_{R \sim R_{0}} \epsilon_{u^{\prime} u 1^{\prime}} X_{u_{1^{\prime} n^{\prime} \alpha^{\prime}}}\right\}\left(B_{j n \alpha^{\prime}}{ }_{j n_{1} \alpha_{1}}+B_{j n 1 \alpha_{1}}+B_{j n \alpha}\right) .
\end{aligned}
$$

The transformation (10) which diagonalizes the Hamiltonian (5) also diagonalizes (33) to give

$$
H_{\mathrm{ex}}=\sum_{I} \omega_{I}\left(B_{I}^{+} B_{I}+\frac{1}{2}\right) \quad \text { with } \quad I=(j, \mathbf{k}, f),
$$

where

$$
\omega_{I}=\omega_{I}(0)+\Delta_{I}(\epsilon)
$$

in which $\omega_{I}(0)$ is the eigenvalue in a lattice without thermal expansion and $\Delta_{I}(\epsilon)$ is the energy correction due to thermal expansion and depends on the strain $\epsilon$ which is temperature dependent. $\Delta_{I}(\epsilon)$ is given by the following 
equation:

$$
\begin{aligned}
\Delta_{I}(\epsilon) & =\sum_{u_{1} u_{2}}\left[\sum_{a, \alpha_{1}, \alpha_{2}}\left(\partial D_{j 0 \alpha_{1}} / \partial R_{u_{1} \alpha \alpha_{1}}\right)_{R=R_{0}} X_{u_{2} \alpha \alpha_{1}} A_{\alpha f} A_{\alpha f}+\sum_{a, \alpha, \alpha_{1}}\left(\partial M_{j, 0 \alpha, c \alpha_{1} /} / \partial R_{u_{1} a \alpha_{1}}\right)_{R=R_{0}}\right. \\
& =\sum_{u_{1} u_{2}} \omega_{j \mathrm{k} f}\left(u_{1} u_{2}\right) \epsilon_{u_{1} u_{2} .}
\end{aligned}
$$

Here $\mathbf{a}$ is the unit cell vector.

To get the explicit temperature dependence, we have to calculate the thermal strain $\epsilon_{u_{14} 2_{2}}$. The thermal strain is determined by the condition that in the absence of any external stress ${ }^{31}$

$$
\left(\partial F / \partial \epsilon_{u_{1} u_{2}}\right)_{T}=0 \text {, }
$$

where $F$ is the Helmholtz free energy of the crystal. It is the sum of a static lattice contribution and a dynamic contribution and is thus given as $F=F_{S}+F_{d}$, where $F_{S}$ is the static contribution while $F_{d}$ is the dynamic contribution. To second order of strain the static lattice contribution is given as

$$
F_{S}=V_{0}+\frac{1}{2} \bar{V} \sum_{u u^{\prime}, u_{1} u_{1}^{\prime}} C_{u u^{\prime} u_{1} u_{1^{\prime}}} \epsilon_{u_{u^{\prime}}} \epsilon_{u_{1} u_{1^{\prime}}}
$$

In this equation $\bar{V}$ is the volume of the crystal in the configuration which corresponds to the minimum of the potential energy and $C_{u u^{\prime} u_{1} u_{1^{\prime}}}$ are the components of the fourth rank elastic tensor..$^{31}$

The dynamic part of the free energy corresponding to the harmonic phonon motions is given by

$$
F_{d}=-(1 / \beta) \ln Z_{d}, \quad \beta=(k T)^{-1},
$$

where $Z_{d}$ is the partition function of the dynamic lattice and is given as

With this expression

$$
Z_{d}=\prod_{\mathrm{q}, S} \sum_{n_{\mathrm{q}} s} \exp \left\{-\beta \omega_{\mathrm{q} S}\left(n_{\mathrm{q} S}+\frac{1}{2}\right)\right\} .
$$

$$
F_{d}=(1 / \beta) \sum_{\mathrm{q}, S} \ln \left[2 \sinh \left(2^{-1} \beta \omega_{\mathrm{q} S}\right)\right]
$$

We now expand $F_{d}$ up to the first order term in the strain and obtain

where

$$
F_{d}=F_{d}{ }^{0}+\sum_{u u^{\prime}} F_{u u^{\prime}} \epsilon_{u u^{\prime}}
$$

$$
F_{u u^{\prime}}=\left(\partial F_{d} / \partial \epsilon_{u u^{\prime}}\right)_{\epsilon=0}=\sum_{\mathbf{q}, S} 2^{-1}\left(2 n_{\mathrm{q} S}+1\right)\left(\partial \omega_{\mathrm{q} S} / \partial \epsilon_{u u^{\prime}}\right)_{\epsilon=0}
$$

To determine $\left(\partial \omega_{\mathrm{q} S} / \partial \epsilon_{u u^{\prime}}\right)_{\epsilon=0}$ we expand the crystal potential energy in the total displacement operators $R_{u n \alpha}$ given by Eq. (24) and retain terms quadratic in $R_{\text {un } \alpha^{0}}$. We then have

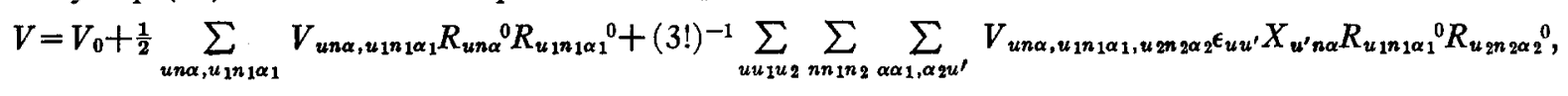

where

$$
V_{u n \alpha, u_{1 n_{1} \alpha_{2}}}=\left(\partial^{2} V / \partial R_{u n \alpha} \partial R_{u_{1 n_{1} \alpha_{1}}}\right)_{0}
$$

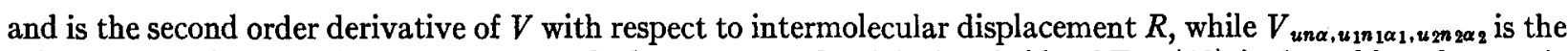
third order derivative with respect to $R$. The last term on the right hand side of Eq. (42) is the cubic anharmonic term. Now if we substitute the expansion (15) for $R_{u n \alpha}{ }^{0}$ and solve the equation of motion, we see that the anharmonic term of Eq. (42), which is linear in the strain $\epsilon$ (the last term on the right hand side) gives a correction $\Delta \omega_{\mathbf{q} S}$ to the eigenvalue $\omega_{\mathbf{q} S}$ of a phonon mode $S$, q. This correction is given as

where

$$
\Delta \omega_{\mathrm{q} S}=\left(2 \omega_{\mathrm{q} S}\right)^{-1} \sum_{u u^{\prime}, u_{1}, u_{2}} d_{u u^{\prime}, u_{1 u_{2}}}(q, S) \epsilon_{u_{u^{\prime}}},
$$

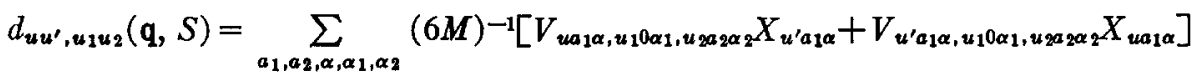

$$
\begin{aligned}
& \times \eta_{\alpha_{1}}{ }^{u_{1}}(\mathbf{q}, S) \eta_{\alpha_{2}}{ }^{u_{2}}(\mathbf{q}, S) \exp (i \mathbf{q} \cdot \mathbf{a}) .
\end{aligned}
$$

Thus

$$
\left(\partial \omega_{\mathrm{q} S} / \partial \epsilon_{u u^{\prime}}\right)_{\epsilon=0}=\left(2 \omega_{\mathrm{q} S}\right)^{-1} \sum_{u_{1}, u_{2}} d_{u u^{\prime}, u_{1} u_{2}}(\mathrm{q}, S) .
$$

Substituting this relation in Eq. (41) we get

$$
F_{u u^{\prime}}=\sum_{\mathrm{q}, S} \frac{1}{4}\left[\left(2 n_{\mathrm{q} S}+1\right) / \omega_{\mathrm{q} S}\right] \sum_{u_{1 u_{1}}} d_{u u^{\prime}, u_{1} u^{\prime}}(\mathrm{q}, S) .
$$


The equation of state given by Eq. (37) becomes

$$
\left(\partial / \partial \epsilon_{u u^{\prime}}\right)\left(F_{S}+F_{d}\right)=0=F_{u u^{\prime}}+\bar{V} \sum_{u_{1 u_{1}^{\prime}}} C_{u u^{\prime} u_{1} u_{1^{\prime}}}
$$

and the formal solution of this equation is

$$
\epsilon_{u u^{\prime}}=-\sum_{\mathbf{q}, S}\left[\left(2 n_{\mathrm{q} S}+1\right) / 4 \omega_{\mathbf{q} S}\right] T_{u u^{\prime}},
$$

where

$$
T_{u u^{\prime}}=(1 / \bar{V}) \sum_{u_{1} u_{1}, u_{2 u z^{\prime}}} C_{u u^{\prime} u 1 u^{1^{\prime}}}{ }^{-1} d_{u_{u^{\prime} u 2 u 2^{\prime}}}
$$

The result, when substituted in Eq. (36), yields

$$
\Delta_{I}(\epsilon)=-\sum_{L} \sum_{u_{1}, u_{2}}\left[\left(2 n_{L}+1\right) / \omega_{L}\right] T_{u_{1} u_{2}} \omega_{I}\left(u_{1} u_{2}\right) \text {, }
$$

where $\omega_{r}\left(u_{1} u_{2}\right)$ is defined by Eq. (36). It can be seen $^{32}$ that this correction has a sign opposite to that of the gas-to-crystal frequency shift and, depending on this, it can show a positive or negative temperature dependence which is described by the phonon occupation number $n_{L}$. This is obviously the result expected from any naive picture in which crystallization is equated to compression and cooling of the crystal with further compression.

\section{SUMMARY OF THEORY}

The results of the above theoretical discussion can be summarized as follows:

(1) The temperature dependence of an intramolecular mode in the harmonic approximation (where a separation between the intramolecular coordinates and the intermolecular coordinate is assumed) is through the (anharmonic) temperature dependence of the lattice frequency and is, thus, an indirect one compared to that of the direct anharmonic interaction where the temperature dependence is due to the presence of the phonon occupation number in the self-energy term. The temperature dependence in the harmonic approximation is thus expected to be small.

(2) The anharmonic contribution to the temperature dependence of a vibrational exciton can be divided in two classes: (i) due to the exciton-phonon interaction, which exists even in a lattice without thermal expansion property, (ii) due to the thermal expansion of the lattice.

(3) The macroscopic theory of the temperature dependence is thus inadequate because it considers only the contribution due to the thermal expansion of the lattice.

(4) Whenever there is a large shift in the frequency of an intramolecular mode in going from the gas phase to the crystal phase, one might expect the frequency to be temperature dependent. It must, however, be remembered that the contribution from the crystal shift constitutes only a part of the total self-energy of the intramolecular mode [see discussion following Eq. (26)] and that in some cases it may happen to have a sign opposite to that of other terms (which might also be significant) in the self-energy. In such cases predictions based only on crystal shift considerations may be misleading.

(5) The temperature dependence of an intramolecular mode should be more pronounced for a low frequency mode and should decrease with the increase in the frequency of the intramolecular mode. This is because its frequency appears in the denominator of the expression for the self-energy.

\section{EXPERIMENTAL}

Durene- $h_{14}$ and durene- $d_{14}$ (Merck, Sharp, and Dohme) were purified by repeated zone refining. For the solution spectra, durene solution in benzene was prepared and used in a capillary. For the crystal work the single crystals were grown from the melt in a capillary under vacuum. The capillary (which remained under vacuum) was used for the experiments. To get a temperature of $100^{\circ} \mathrm{K}$, the sample (capillary) was cooled by a flow of nitrogen vapor. The flow of helium vapor provided a lower temperature $\left(25^{\circ} \mathrm{K}\right)$. The spectra in benzene solution and in the crystalline phase at room temperature, 100 , and $25^{\circ} \mathrm{K}$ were recorded photoelectrically on a Ramalog-Spex double spectrometer model 1401 using direct current. The excitation was provided by an argon ion laser from coherent radiation laboratory. The $4880 \AA$ lasing emission selected by a suitable interference filter was used for the excitation. The spectral resolution was about $1 \mathrm{~cm}^{-1}$.

\section{RESULTS AND DISCUSSION}

Figure 1 displays the region $0-360 \mathrm{~cm}^{-1}$ of the durene$h_{14}$ spectra. The spectra have been taken under four different conditions. A represents the spectrum in the benzene solution..$^{33} \mathrm{~B}$ is the spectrum taken with the durene- $h_{14}$ neat crystal at room temperature. C and D are, respectively, the neat crystal spectra at 100 and $25^{\circ} \mathrm{K}$. A vibrational analysis of this region based on isotopic studies is reported separately. ${ }^{30}$ The transitions at 48,87 , and $114 \mathrm{~cm}^{-1}$ in the neat crystal spectrum at $100^{\circ} \mathrm{K}$ are due to librational phonons ${ }^{30}$ (previously we have called them librational excitons ${ }^{22}$ ). They are absent in the benzene solution spectrum. The change in their frequencies from room temperature to $100^{\circ} \mathrm{K}$ is about $11 \%$. The change from 100 to $25^{\circ} \mathrm{K}$ is small. The transitions at 193 and $163 \mathrm{~cm}^{-1}$ in the crystal spectrum at $100^{\circ} \mathrm{K}$ are due to the methyl torsions. ${ }^{30}$ We have not been able to locate them in the benzene solution spec- 


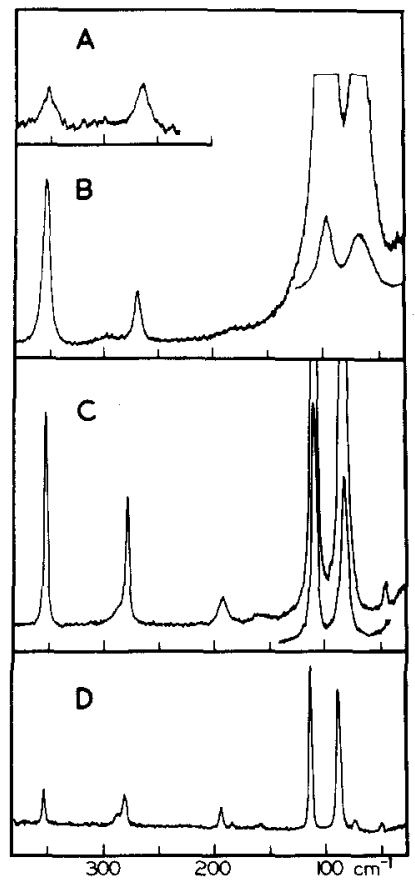

FIG. 1. Raman spectra of durene- $h_{14}$ in the region $0-360$ $\mathrm{cm}^{-1}$ at a resolution of $1 \mathrm{~cm}^{-1}$. $A$ represents the spectrum in the benzene solution. $\mathrm{B}, \mathrm{C}$, and $\mathrm{D}$ are, respectively, durene- $h_{14}$ neat crystal spectra at room temperature, 100 , and $25^{\circ} \mathrm{K}$.

trum. Even at room temperature these are too broad (and weak?) to locate their exact peak position. The transition at $281 \mathrm{~cm}^{-1}$ in the $100^{\circ} \mathrm{K}$ spectrum is because of an intramolecular mode which involves the in-plane scissor-like displacement of the entire methyl group with respect to the aromatic ring. ${ }^{34}$ This is the intramolecular mode which is sensitive to the temperature. We call this mode $I$. We have not been able to get the durene spectrum in the vapor phase, but the spectrum in dilute benzene solution ${ }^{33}$ should give us a measure of the crystal shift and its direction (positive or negative). The frequency of this mode in benzene solution is $265 \mathrm{~cm}^{-1}$ and thus the shift from the benzene solution to the crystal phase is $7 \mathrm{~cm}^{-1}$ to higher energy. ${ }^{33}$ This frequency further shifts from room temperature to $100^{\circ} \mathrm{K}$ by $9 \mathrm{~cm}^{-1}$. This temperature shift is about $3.3 \%$. Below $100^{\circ} \mathrm{K}$ the temperature dependence, again, is small. The transition at $354 \mathrm{~cm}^{-1}$ in the crystal spectra is due to another intramolecular fundamental. Though the shift in its frequency from the benzene solution to the room temperature crystal is $3 \mathrm{~cm}^{-1}$ to higher energy, this mode does not show any temperature dependence in its frequency.

Figure 2 shows a completely analogous situation in durene- $d_{14}$. The frequency shift of the phonon transitions from room temperature to $100^{\circ} \mathrm{K}$ is again about $11 \%$. The mode at $250 \mathrm{~cm}^{-1}$ in the crystal spectrum at $100^{\circ} \mathrm{K}$ corresponds to band $I$ at $281 \mathrm{~cm}^{-1}$ in the $100^{\circ} \mathrm{K}$ crystal spectrum of durene- $h_{14}$. This band shifts by $8 \mathrm{~cm}^{-1}$ to higher energy in going from dilute benzene solution $^{33}$ to the room temperature crystal phase. The change from room temperature to $100^{\circ} \mathrm{K}$ is, again, $8 \mathrm{~cm}^{-1}$.

The results of the present investigation are summarized in Table I. Below we attempt to relate them to anharmonic interactions.
The direction of the shift from the benzene solution to the crystal phase indicates that the crystal shift is positive (i.e., there is an increase in the frequency in going to crystal phase). On the other hand, the shift from room temperature to low temperature shows a negative temperature dependence (i.e., there is an increase in the frequency with a decrease in the temperature). This is what would be expected from the contribution due to thermal expansion of the lattice for this case where the crystal shift is positive (to higher energy). However, in view of the fact that the shift from dilute benzene solution ${ }^{33}$ to the room temperature crystal spectrum is smaller than the temperature shift in the case of durene- $h_{14}$ and equal to the temperature shift in the case of durene- $d_{\mathbf{1 4}}$, it does not seem very likely that this temperature shift is entirely (or even mainly) due to the thermal expansion and, thus, we have to look for other causes [class (i)] discussed earlier. The quartic anharmonicity contribution seems to be small as it would give rise to a positive temperature dependence in this case (because of positive crystal shift). As the frequency of this mode $I$ is greater than twice the maximum lattice frequency, in the case where $L_{1}$ and $L_{2}$ are both lattice modes, the first term on the right hand side of Eq. (27) will show a positive temperature dependence and is thus not responsible for the observed temperature dependence of the mode $I$. On the other hand, the anharmonic term $V_{I T_{1} L_{2}}$ in which $T_{1}$ is a methyl torsional mode and $L_{2}$ is a lattice mode can give rise to a negative temperature dependence for durene- $h_{14}$, as $\left(\omega_{T_{1}}+\omega_{L_{2}}\right)$ in such a case will be greater than $\omega_{I}$ (see the data at $100^{\circ} \mathrm{K}$ ). This, however, is not the case with durene- $d_{\mathbf{1 4}}$ where, in a similar mechanism,

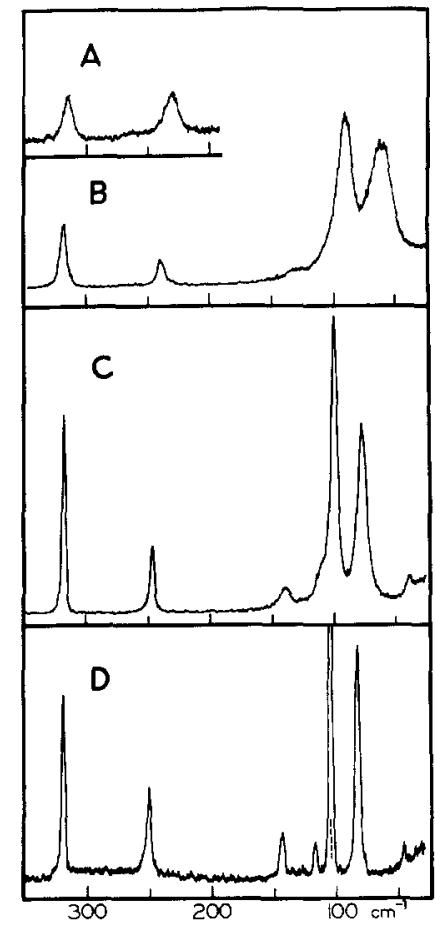

Fig. 2. Raman spectra of durene- $d_{14}$ in the region 0 $350 \mathrm{~cm}^{-1}$ at a resolution of $1 \mathrm{~cm}^{-1}$. A represents the spectrum in the benzene solution. $\mathrm{B}, \mathrm{C}$, and $\mathrm{D}$ are, respectively, durene- $d_{14}$ neat crystal spectra at room temperature, 100 , and $25^{\circ} \mathrm{K}$. 
TABle I. Durene Raman frequencies (in $\mathrm{cm}^{-1}$ ) in the region $0-360 \mathrm{~cm}^{-1}$.

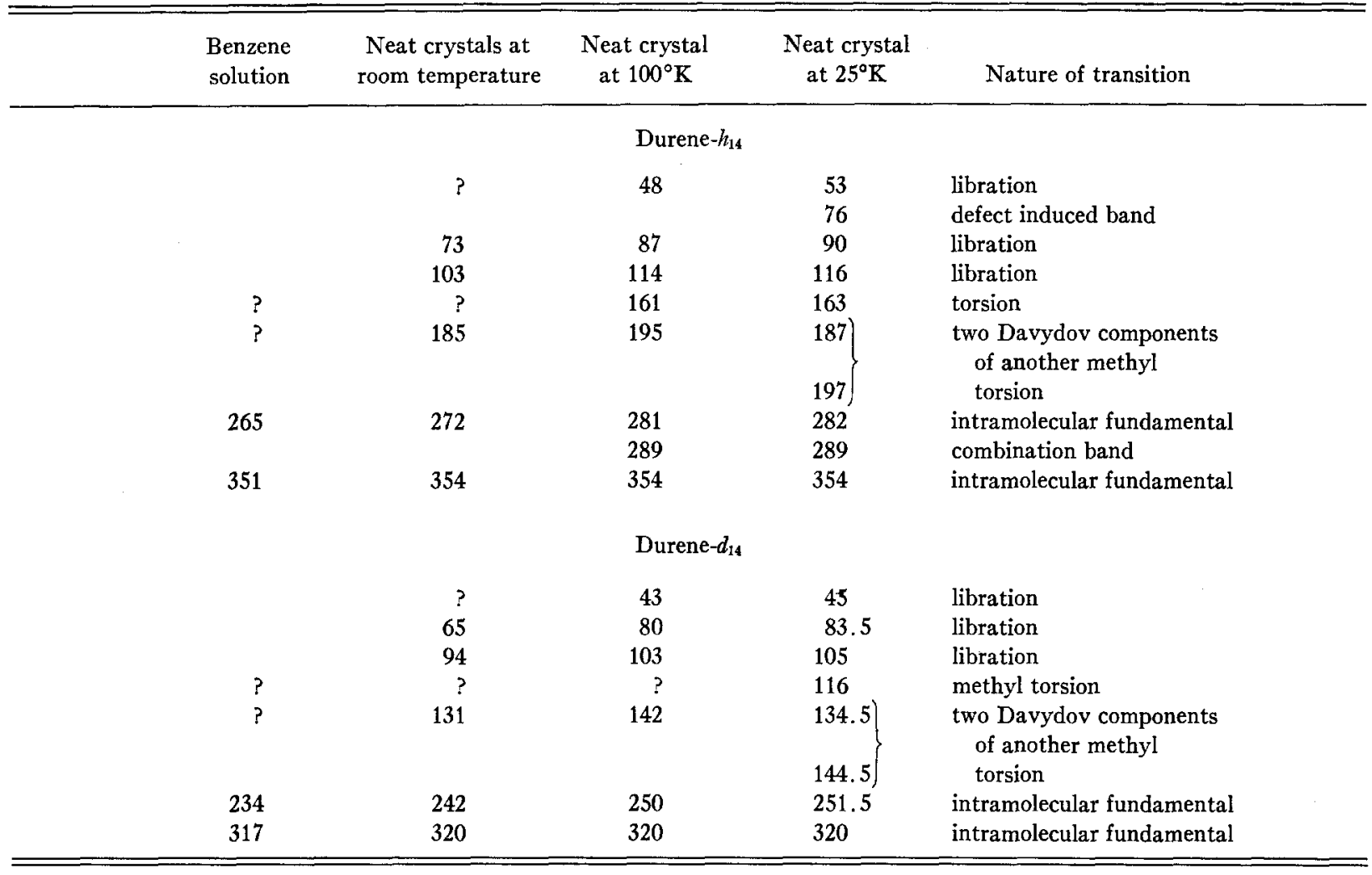

$\left(\omega_{T_{1}}+\omega_{L_{2}}\right)<\omega_{I}$. As the temperature dependence in both cases is quite similar, this mechanism cannot be responsible for the observed temperature dependence. Another possible mechanism is the one where $L_{1}$ and $L_{2}$ are both methyl torsions $T_{1}$ and $T_{2}$ (like the 163 and $193 \mathrm{~cm}^{-1}$ modes in durene- $\left.h_{14}\right)$. In this case $\left(\omega_{T_{1}}+\omega_{T_{2}}\right)>$ $\omega_{I}$ for both durene- $h_{14}$ and durene- $d_{14}$. However, as the occupation numbers $n_{T_{1}}$ and $n_{T_{2}}$ for these modes will be small compared to the occupation numbers of the lattice modes, we expect a small contribution from such a mechanism.

The contribution from the second and the third term on the right hand side of Eq. (27) is smaller than the first term because of the large energy denominator.

The fourth term on the right hand side of Eq. (27) gives a positive contribution for all modes $L_{1}$ and $L_{2}$ and may thus, be chiefly responsible for the temperature dependence of the $281 \mathrm{~cm}^{-1}$ vibrational mode of durene$h_{14}$ and the $250 \mathrm{~cm}^{-1}$ mode of durene- $d_{14}$ at $100^{\circ} \mathrm{K}$.

Let us now consider the physical nature of the temperature dependent mode $I$. A normal coordinate analysis $^{29}$ suggests that this mode involves the totally symmetric ( $A_{g}$ in $D_{2 h}$ point group) in-plane scissor-like displacement of the entire methyl group with respect to the benzene ring. Such motion should be very sensitive to the nature of the methyl torsions and the barrier hindering it. A reason for such dependence is that the nonbonded $\mathrm{C}-\mathrm{H}$ and $\mathrm{H}-\mathrm{H}$ interactions of the methyl groups contribute both to this vibration and the methyl torsions. The methyl torsions, on the other hand, are very sensitive to the lattice perturbation. Thus, it seems likely that the temperature dependence should come through the participation of such methyl torsions in the anharmonic interaction with mode $I$. In light of the above theoretical and physical arguments it appears that the observed temperature dependence is, most probably, due to the fourth term of Eq. (27),

$$
-36 V_{I I T_{1}} V_{T_{1} L_{2} L_{2}}\left[\left(2 n_{L_{2}}+1\right) / \omega_{T_{1}}\right],
$$

where $T_{1}$ represents a methyl torsion and $L_{2}$ is a lattice mode.

In conclusion we have shown that in complex molecular crystals, where motions like torsions exist that are intermediate in nature compared to lattice modes on one hand and to intramolecular vibrations (other than torsions) on the other hand, the rigid body assumption of molecular lattice dynamics is, perhaps, not a good approximation. The anharmonic interactions which involve the participation of torsions may show interesting manifestations (like temperature dependent shift and broadening). In such cases a distinction between the lattice modes and the intramolecular modes based on the criterion that only the lattice modes show frequency shift with temperature may lead to erroneous results. We suggest that in these cases our isotopic mixed crystal criterion, ${ }^{35}$ stating that the phonons obey the amalgamation limit in the isotopic mixed crystals whereas in the same system intramolecular vibrations are in the separated band limit, should be more reliable. The amalgamation limit implies a small isotopic per- 
turbation compared to the bandwidth. In this limit the phonon spectrum changes in nearly a linear fashion with concentration of the isotopic component. In the separated band limit, on the other hand, the isotopic perturbation is much larger than the bandwidth and in a $50 \%$ isotopic mixed crystal two transitions corresponding to both the per proto and per deutero compound will appear. This is clearly demonstrated in the case of durene where the temperature dependent intramolec- ular mode as well as the torsions are in the separated band limit, while the phonons exhibit the amalgamation limit. ${ }^{30}$

\section{ACKNOWLEDGMENT}

We thank the National Science Foundation for Grant GP-8345, which enabled the departmental purchase of the Ramalog-Spex double spectrometer.

\section{APPENDIX}

The solution for the self-energy is most conveniently derived by solving the equation of motion for the doubletime retarded exciton Green's function. The double-time retarded Green's function for excitons is defined $a^{36,37}$

$$
G(I ; t)=-i \theta(t)\left\langle\left[B_{I}(t), B_{I}^{+}(0)\right]\right\rangle,
$$

where $\theta(t)$ is a unit step function.

The total Hamiltonian for the vibration is

$$
H=\sum_{I} \omega_{I}\left(B_{I}{ }^{+} B_{I}+\frac{1}{2}\right)+\sum_{L} \omega_{L}\left(b_{L}{ }^{+} b_{L}+\frac{1}{2}\right)+\sum_{I, L} V_{I L}\left(B_{I}+B_{I}^{+}\right)\left(b_{L}+b_{L}{ }^{+}\right) .
$$

The Heisenberg equations of motion for the exciton operators are given as

$$
i\left(d B_{I} / d t\right)=\left[B_{I}, H\right]=\omega_{I} B_{I}+\sum_{L} V_{I L}\left(b_{L}+b_{L}^{+}\right)
$$

and

$$
-i\left(d B_{I}^{+} / d l\right)=\omega_{I} B_{I}^{+}+\sum_{L} V_{I L}\left(b_{L}+b_{L}^{+}\right)
$$

Similarly,

$$
i\left(d b_{L} / d t\right)=\omega_{L} b_{L}+\sum_{I} V_{L I}\left(B_{I}+B_{I}^{+}\right)
$$

and

$$
-i\left(d b_{L}{ }^{+} / d t\right)=\omega_{L} b_{L}++\sum_{T} V_{L I}\left(B_{I}+B_{I}^{+}\right) .
$$

With these values we can derive the equations of motion for the retarded Green's function of the excitons:

$$
i[d G(I ; t) / d t]=\delta(t)+\omega_{I} G(I ; t)+\sum_{L} V_{I L}\left\{G^{1}(I, L ; t)+G^{2}(I, L ; t)\right\},
$$

where

and

$$
G^{\mathbf{1}}(I, L ; t)=-i \theta(t)\left\langle\left[b_{L}(t), B_{I}{ }^{+}(0)\right]\right\rangle
$$

$$
G^{2}(I, L ; t)=-i \theta(t)\left\langle\left[b_{L}+(t), B_{I}^{+}(0)\right]\right\rangle .
$$

The equations of motion for $G^{1}(I, L ; t)$ and $G^{2}(I, L ; t)$ give

and

$$
i\left[d G^{1}(I, L ; t) / d t\right]=\omega_{L} G^{\mathbf{1}}(I, L ; t)+V_{L I} G(I ; t)
$$

$$
i\left[d G^{2}(I, L ; t) / d t\right]=-\omega_{L} G^{2}(I, L ; t)-V_{L I} G(I ; t) .
$$

We now introduce $G^{1}(I, L ; \omega), G^{2}(I, L ; \omega)$, and $G(I, \omega)$, which are Fourier transforms, respectively, of $G^{1}(I, L ; t)$, $G^{2}(I, L ; t)$, and $G(I ; t)$. If we take the Fourier transform of the Eq. (A7) and solve for $G^{1}(I, L ; \omega)$ and $G^{2}(I, L ; \omega)$ we get

and

$$
G^{1}(I, L ; \omega)=V_{L I} G(I ; \omega) /\left(\omega-\omega_{L}\right)
$$

$$
G^{2}(I, L ; \omega)=-V_{L I} G(I ; \omega) /\left(\omega+\omega_{L}\right) .
$$


We then take the Fourier transform of Eq. (A5) and solve for $G(I ; \omega)$ with the help of Eqs. (A8) and (A9). We get

$$
G(I ; \omega)=\left\{\omega-\left[\omega_{I}+\sum_{L}\left(\frac{V_{I L} V_{L I}}{\omega-\omega_{L}}-\frac{V_{I L} V_{L I}}{\omega+\omega_{L}}\right)\right]\right\}^{-1} .
$$

The self-energy (or the energy correction to $\omega_{I}$ ) $\Delta_{I}$ is thus given as

$$
\left.\Delta_{I}=\sum_{L}\left\{\left[V_{I L} V_{L I} P /\left(\omega-\omega_{L}\right)\right]-V_{I L} V_{L I} /\left(\omega+\omega_{L}\right)\right]\right\},
$$

where $P$ ensures that the first term vanishes when $\omega=\omega_{L}$.

\section{* Supported by NSF Grant GP-18718 and NIH Grant} NS08116.

${ }^{1}$ W. Cochran and G. S. Pawley, Proc. R. Soc. A 280, 1 (1964).

${ }^{2}$ H. Hahn and W. Biem, Phys. Status Solidi 3, 1911 (1963).

${ }^{3}$ G. S. Pawley and S. J. Cyvin, J. Chem. Phys. 52, 4073 (1970).

${ }^{4} \mathrm{G}$. Venkataraman and V. C. Sahni, Rev. Mod. Phys. 24, 409 (1970).

${ }^{5}$ G. S. Pawley, Phys. Status Solidi 20, 347 (1967).

${ }^{6}$ G. Dolling and B. M. Powell, Proc. R. Soc. A 319, 209 (1970).

${ }^{7}$ R. Stockmeyer and H. Stiller, Phys. Status Solidi 27, 269 (1968).

${ }^{8}$ H. A. Rafizadeh and S. Yip, J. Chem. Phys. 53, 315 (1970).

${ }^{9}$ R. M. Hexter, J. Chem. Phys. 33, 1833 (1960); J. Chem. Phys. 36, 2285 (1962).

${ }^{10} \mathrm{D}$. Dows, in Physics and Chemistry of the Organic Solid State, edited by D. Fox, M. M. Labes, and A. Weissberger (Interscience, New York, 1963), Vol. II.

${ }^{11}$ R. Kopelman, J. Chem. Phys. 47, 3227 (1967).

${ }^{12}$ G. W. Robinson, Annu. Rev. Phys. Chem. 21, 429 (1970).

${ }^{13} \mathrm{O}$. Schnepp, in Advances in Atomic and Molecular Physics, edited by D. R. Bates and I. Esterman (Academic, New York, 1969).

${ }^{14}$ S. S. Mitra, J. Chem. Phys. 39, 3031 (1963).

${ }^{15}$ L. Liebermann, J. Acoust. Soc. Am. 31, 1073 (1959); Phys. Rev. 113, 1052 (1959).

${ }^{16}$ S. S. Yun and R. T. Beyer, J. Chem. Phys. 40, 2538 (1964)

${ }^{17}$ L. Liebermann, Phys. Rev. 113, 1052 (1959); R. A. Rasmussen, J. Chem. Phys. 36, 1821 (1962).

${ }^{18}$ A. Warshel, J. Chem. Phys. 54, 5324 (1971).

${ }^{19} \mathrm{M}$. Ito, M. Suzuki, and T. Yokoyama, in Excitons, Magnons and Phonons in Molecular Crystals, edited by A. B. Zahlan (Cambridge U. P., London, 1968), p. 1.

${ }^{20}$ A. Hadni, B. Wyncke, G. Morlot, and X. Gerbaux, J. Chem. Phys. 51, 3514 (1969).

${ }^{21}$ This is derived from the fact that for the lowest intramolecular mode (except for torsions) the crystal shift is positive. If the terms like $V_{j j^{\prime}, n}$ a make the major contribution, the crystal shift should be negative because of the negative energy denominator.

${ }^{22}$ Recently the concept of librational exciton has been introduced by us, see P. N. Prasad and R. Kopelman, J. Chem. Phys. 57, 863 (1972). However, in the present context where we are considering the role of a lattice vibration in general (and not just librations), the term phonon is more appropriate. This also serves the purpose of distinguishing them from the intramolecular mode.

${ }^{23}$ For this reason the approximation where only the anharmonic interaction of class (ii) is included but not that of (i) is known as quasiharmonic approximation. This is because the correction of class (ii) can be incorporated in the harmonic approximation by a simple canonical transformation.
${ }^{24}$ The derivation of the expression for the anharmonic self-energy of an intramolecular mode follows a course similar to that for a localized lattice mode. The latter has been discussed at many places. See A. A. Maradudin, Solid State Phys. 19, 1 (1966), and the references therein. The derivation is most conveniently accomplished by solving the equations of motion for the Green's function. See the Appendix.

${ }^{25}$ Here the separability between the intramolecular coordinate and the intermolecular coordinate has been assumed. This assumption is sometimes called "double adiabatic approximation." In the double adiabatic approximation, after separating the electronic motion from the vibrational motion using the adiabatic approximation, the latter is used again to separate one vibrational subsystem (intramolecular motion) from the other (lattice motion). See K. K. Rebane, Impurity Spectra of Solids, translated by John S. Shier (Plenum, New York, 1970).

${ }^{26}$ R. M. Hochstrasser and P. N. Prasad, Chem. Phys. Lett. 8, 315 (1971).

${ }^{27}$ R. M. Hochstrasser and P. N. Prasad, J. Chem. Phys. 56, 2814 (1972).

${ }^{28}$ In a simple crystal potential model the odd derivatives of the crystal potential with respect to the intermolecular displacement $(R)$ should have the same sign. Thus $V_{I L}$ which involves the first derivative of the crystal potential ( $D$ and $M$ terms in this case) with respect to $R$, should have the same sign as $V_{L_{1} L_{2} L_{2}}$ (which is a third derivative with respect to $R$ ) if $D$ and $M$ or the dominant of the two are positive.

${ }^{29}$ Again, in a simple potential model, the even (quadratic, in this case) derivative of the crystal shift terms will have the same sign as that of the crystal shift terms.

${ }^{30}$ P. N. Prasad and R. Kopelman, J. Chem. Phys. 58, 126 (1973)

${ }^{31}$ Our approach is similar to that taken by Maradudin for a lattice mode. See A. A. Maradudin, Phys. Status Solidi 2, 1493 (1962).

${ }^{32}$ This is based on the fact that $\omega_{I}\left(u_{1} u_{2}\right)$ and $d_{u u^{\prime} u_{2} u_{2}^{\prime}}$ (which is contained in $T_{u_{1} u_{2}}$ ) are both odd derivatives with respect to the intermolecular displacements. They have the same sign if $D$ and $M$ are positive. See Footnote 28.

${ }^{33}$ We have also taken the spectrum of a dilute solution of durene in carbon tetrachloride. The frequency of this transition is the same (within $\pm 1 \mathrm{~cm}^{-1}$ ) in the two solvents. This indicates to us that the solvent shifts are small.

${ }^{34}$ M. A. Kovner, Opt. Spektrosk. 1, 348 (1956).

${ }^{35}$ P. N. Prasad and R. Kopelman, J. Chem. Phys. 57, 856 (1972); J. Chem. Phys. 57, 863 (1972).

${ }^{36}$ D. N. Zubarev, Sov. Phys.-Usp. 3, 320 (1960).

${ }^{37}$ A. S. Davydov, Theory of Molecular Excitons, translated by B. Dresner (Plenum, New York, 1971). 